\title{
MULTILAYER NEUTRON MIRRORS AND THEIR APPLICATIONS
}

\author{
T. KAWAI
}

Research Reactor Institute Kyoto University, Kumatori-cho, Osaka 590-04, Japan

The purpose of this report is to talk, in an instructive way, about multilayer neutron mirrors and their applications developed in our institute. The outline is as follows. Basic properties and the reflectivity of the multilayer neutron mirror are given. The applications of the multilayer neutron mirrors, i.e., multilayer neutron monochromators, multilayer neutron polarizers, supermirrors, are presented. Measuring methods of the reflectivity and the polarization are secondly reported.

PACS numbers: 07.60.-j

\section{Introduction}

The wave property as de Broglie wave determined from mass and velocity is drawn out conspicuously by combining cold neutrons with neutron mirrors. A neutron mirror is a neutron optical device utilizing the total reflection from the interface between vacuum and a coherently scattering medium. When a neutron wave propagates in a medium, the spherical waves scattered from individual atoms interfere with a propagating plane wave. This causes a change of the wave vector of the incident neutron wave. Now let us consider $k_{0}$ as a neutron wave vector in the vacuum and $k$ - in the medium. The ratio $n=k / k_{0}$ is called the refractive index, since a change in the wave vector leads to refraction of the wave at the boundary between the vacuum and the medium according to Snell's law

$$
n=\frac{k}{k_{0}}=\frac{\cos \theta_{0}}{\cos \theta} \text {. }
$$

Many kinds of materials have the refractive index less than unity, and thus are optically less dense than vacuum. When a neutron wave is incident on an optically less dense medium, the total reflection occurs if the incident angle $\theta_{0}$ is smaller than some critical angle $\theta_{c}[1-6]$. The critical angle $\theta_{c}$ is determined from the condition, $n=\cos \theta_{c} \simeq 1-\theta_{c}^{2} / 2$, since $\theta_{c} \ll 1$.

The neutron guide tubes utilizing the total reflection of the $\mathrm{Ni}$ mirror are used for extracting thermal and cold neutrons to a distant place without loss of intensity in many institutes [7-10].

A multilayer neutron mirror is an artificial lattice with a large interplanar spacing and gives the simple one-dimensional optical potential to neutrons, which is related to the refractive index of the mirror material [11-13]. The width and height of the potential are controlled arbitrarily. The potential width is determined 
by the distribution of layer thicknesses during vacuum evaporation and the height by selecting layer materials [14]. Ferromagnetic materials produce magnetic optical potentials, and are used as neutron magnetic mirrors for controlling the static or dynamic spin states of neutron as a polarizer or a pulser [15].

Monochromator consisting of alternating parallel layers of two substances with different refractive indices (coherent scattering lengths) but equal thickness is used for extracting neutrons of the definite wavelength [16, 17]. This device uses the Bragg reflections like in the case of crystals. Polarizing monochromator is fabricated with ferromagnetic materials [18].

By changing slowly the period of multilayer structures, the effective reflection angle could be larger than the total reflection angle. This idea was realized by Mezei [19] for extracting thermal neutrons with a sufficiently wide wavelength range. Such devices are called supermirrors. A usual supermirror is consisting of a total reflection mirror and a so-called supermirror. The supermirror guide tube was firstly constructed at B-4 beam hole of KUR in 1985 [9].

Taking one's attention to the fact that the reflectivity depends on refractivities of materials, the distribution of substances is obtained from the measurement of neutron reflectivity from the specular sample [20]. The neutron reflectometer is used now for studying the structure of the composite polymers [21-23]. A micro neutron-interferometer consisting of a thin palladium film sandwiched by two semitransparent multilayer mirrors is used for determining the hydrogen content in the evaporated palladium film [24].

\section{Cold neutrons and multilayer neutron mirrors}

Cold neutrons are commonly defined as those having wavelengths longer than $4 \AA$ which corresponds to the Bragg cutoff in beryllium [5]. The intensity of cold neutrons is, however, about $1 \%$ or $2 \%$ of the total neutron flux in the thermal distribution in a conventional research reactor, or a spallation neutron source. Thus, the cold neutron source of which a moderator is liquid hydrogen or liquid deuterium of about $20 \mathrm{~K}$, is installed near by the neutron source for increasing the cold neutron flux [25].

Neutrons with wavelengths shorter than $1 \AA$ show the corpuscular property and could be dealt classically. A cold neutron exhibits, however, the wave property as de Broglie wave remarkably and thus is understood with wave mechanics.

We consider the coherent elastic scattering of a neutron represented by a plane wave from the boundary between vacuum and material medium and assume the specular reflection. This is satisfied under the condition that the glancing angle of incidence $\theta_{0}$ of incident wave vector $k_{0}$ is the same as the reflected angle $\theta$ of the wave vector $k$. When a neutron is reflected from the specular surface, neutron reflectivity is a function of a wave vector transfer $Q$ which is perpendicular to the boundary. The magnitude of the wave vector transfer is given by $|Q|=\left|k-k_{0}\right|=$ $2 k_{0} \sin \theta_{0}$, where $|k|=\left|k_{0}\right|=k_{0}=2 \pi / \lambda$.

The potential energy of neutron in the vacuum is zero and the kinetic energy is equal to $\hbar^{2} k_{0}^{2} / 2 m$, where $\hbar$ is Planck's constant divided by $2 \pi$ and $m$ is its mass. In a medium, the total energy $E$ is given by 


$$
E=\frac{\hbar^{2} k^{2}}{2 m}+V
$$

where $k$ is the neutron wave vector in the medium. The effective potential of medium $V$ is expressed in terms of a coherent scattering length $b_{\mathrm{N}}$

$$
V=\frac{2 \pi \hbar^{2} b_{\mathrm{N}} \rho}{m}
$$

This potential is called Fermi potential. The neutron total energy inside and outside the medium is the same so that we obtain

$$
\frac{k}{k_{0}}=\left(1-\frac{2 m V}{\hbar^{2} k_{0}^{2}}\right)^{1 / 2}=\left(1-\frac{4 \pi b_{\mathrm{N}} \rho}{k_{0}^{2}}\right)^{1 / 2} \simeq 1-\frac{\lambda^{2} b_{\mathrm{N}} \rho}{2 \pi} \equiv n,
$$

where $\lambda$ is the incident neutron wavelength, $b_{N}$ - the nuclear coherent scattering length and $\rho$ - the number of atoms per unit volume. The refractive index $n$ takes an imaginary value if $1-4 \pi b_{\mathrm{N}} \rho / k_{0}^{2}<0$.

Since $\theta_{\mathrm{c}} \ll 1, \cos \theta_{\mathrm{c}}$ can be expanded and we obtain

$$
\theta_{\mathrm{c}}=\sqrt{2(1-n)}=\lambda \sqrt{\frac{b_{\mathrm{N}} \rho}{\pi}} \text {. }
$$

Let us take $E_{0}$ as energy of the incident neutron. The critical angle is given as $\theta_{c}=\left(V / E_{0}\right)^{1 / 2}$. The normal component of the wavelength, satisfying the condition that the incident angle is equal to the critical angle of the total reflection, is called the critical wavelength, $\lambda_{c}$, i.e., $2 \pi / \lambda_{c}=k_{0} \sin \theta_{c}=\sqrt{4 \pi b_{N} \rho}$.

We thus obtain the coherent scattering length by measuring the critical angle for a definite wavelength.

If the mirror is made of polished magnetized ferromagnetic material, the magnetic optical potential, $V_{\mathrm{m}}$, is introduced into the formula for the refractive index [26]

$$
V_{\mathrm{m}}=-\mu B= \pm \mu B= \pm 6 \times 10^{-12} B[\mathrm{eV}]
$$

where $\mu$ is the magnetic moment of the neutron, $B-$ the saturated magnetic induction. The plus and minus signs refer to neutron spin parallel and antiparallel to the direction of magnetization of the magnetic mirror. The magnetic scattering of neutrons occurs because of the interaction between the magnetic moment of the neutron and that of the atom, while nuclear scattering occurs on the nuclei of material.

The critical angle is now given by the formula

$$
\theta_{\mathrm{c}}=\lambda \sqrt{\frac{b_{\mathrm{N}} \rho}{\pi} \pm \frac{m \mu B}{2 \pi \hbar^{2}}} .
$$

Now we take $V_{\mathrm{N}}$ as a nuclear scattering potential. If

$$
V_{\mathrm{m}} \geq V_{\mathrm{N}}
$$

$n_{-} \geq 1$ and $n_{+}<1$ are obtained. In this case, the neutrons with spins parallel to the direction of the magnetization of the mirror are reflected, while neutrons with the antiparallel spin direction are transmitted.

Let the $x$ axis be the direction normal to the surface of the multilayer and the neutron is incident on the surface from the $x$ direction. Thus $k$ and the potential are 
functions of only $x$. The time independent Schrödinger equation, which describes the propagation of the neutron wave and its reflection at the multilayer, is

$$
\psi^{\prime \prime}(x)+k_{x}^{2}(x) \psi(x)=0,
$$

where

$$
k_{x}^{2}=k_{0 x}^{2}\left(1-\frac{4 \pi b_{\mathrm{N}} \rho}{k_{0 x}^{2}}\right)=k_{0 x}^{2} n_{x}^{2} .
$$

The solution of Eq. (10) for the reflection of neutron from the multilayer mirror was solved by the method analogous to the conventional optics $[11,13]$ when the potential could be divided into a discrete number of rectangular potentials as shown in Fig. 1.

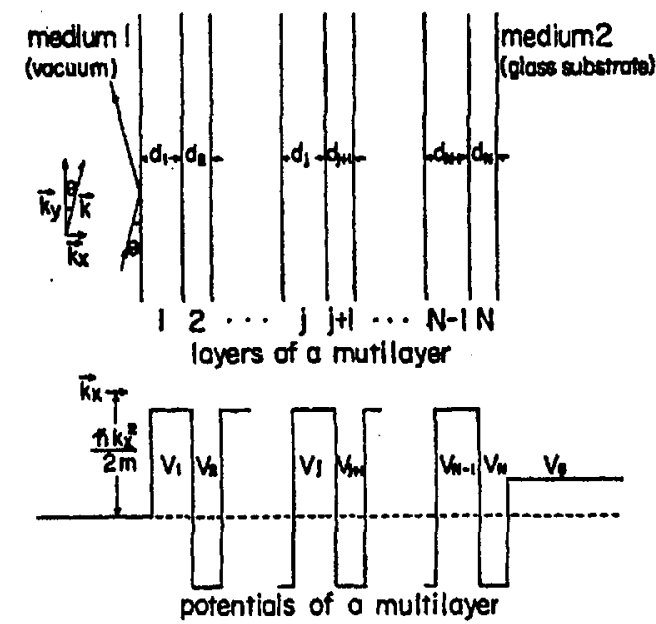

Fig. 1. The wave vector of the incident neutron and stacked assembly of one-dimensional square well potentials of a multilayer.

The reflectivity and transmission amplitudes, $R$ and $T$, are obtained by imposing the conditions that at boundaries of layers the wave function in the $j$-th layer

$$
\psi_{j}(x)=A_{j} \exp \left(\mathrm{i} k_{j x} x\right)+B_{j} \exp \left(-\mathrm{i} k_{j x} x\right)
$$

and its first derivative must be continuous. $j$ means the $j$-th layer and $k_{j x}$ is the normal component of wave vector in the $j$-th layer. Hereafter, we omit the suffix $x$

$$
\begin{aligned}
& \psi_{j}\left(x_{j}\right)=\psi_{j+1}\left(x_{j}\right), \\
& \psi_{j}^{\prime}\left(x_{j}\right)=\psi_{j+1}^{\prime}\left(x_{j}\right) .
\end{aligned}
$$

The simultaneous equations for amplitudes, $A, B$, are obtained in the matrix notation

$$
\begin{aligned}
& \left(\begin{array}{cc}
\exp \left(\mathrm{i} k_{j} x_{j}\right) & \exp \left(-\mathrm{i} k_{j} x_{j}\right) \\
\mathrm{i} k_{j} \exp \left(\mathrm{i} k_{j} x_{j}\right) & -\mathrm{i} k_{j} \exp \left(-\mathrm{i} k_{j} x_{j}\right)
\end{array}\right)\left(\begin{array}{c}
A_{j} \\
B_{j}
\end{array}\right)= \\
& \left(\begin{array}{cc}
\exp \left(\mathrm{i} k_{j+1} x_{j}\right) & \exp \left(-\mathrm{i} k_{j+1} x_{j}\right) \\
\mathrm{i} k_{j+1} \exp \left(\mathrm{i} k_{j+1} x_{j}\right) & -\mathrm{i} k_{j+1} \exp \left(-\mathrm{i} k_{j+1} x_{j}\right)
\end{array}\right)\left(\begin{array}{c}
A_{j+1} \\
B_{j+1}
\end{array}\right),
\end{aligned}
$$




$$
\left(\begin{array}{c}
\exp \left(\mathrm{i} k_{0} x_{0}\right)+r \exp \left(-\mathrm{i} k_{0} x_{0}\right) \\
\mathrm{i} k_{0} \exp \left(\mathrm{i} k_{0} x_{0}\right)-\mathrm{i} k_{0} r \exp \left(-\mathrm{i} k_{0} x_{0}\right)
\end{array}\right)=M\left(\begin{array}{c}
t \exp \left(\mathrm{i} k_{N+1} x_{N}\right) \\
\mathrm{i} k_{N+1} t \exp \left(\mathrm{i} k_{N+1} x_{N}\right)
\end{array}\right) \text {, }
$$

where we assume that the amplitude of incident wave is 1 and that of reflected one from the surface of the multilayer is $r$. The amplitude of transmitted wave is $t$ and that of reflected one 0 in the $(N+1)$-th layer, i.e., in the exit side of the multilayer mirror. The characteristic matrix $M$ is given as follows

$$
\begin{aligned}
& M=M_{1} M_{2} \cdots M_{N-1} M_{N}, \\
& M_{j}=\left(\begin{array}{cc}
\cos \left(k_{j} d_{j}\right) & -\frac{1}{k_{j}} \sin \left(k_{j} d_{j}\right) \\
k_{j} \sin \left(k_{j} d_{j}\right) & \cos \left(k_{j} d_{j}\right)
\end{array}\right),
\end{aligned}
$$

where $d_{j}$ is a thickness of $j$-th layer. A reflectivity and a transmissivity of the multilayer mirror are thus obtained

$$
\begin{aligned}
& R=|r|^{2}, \\
& T=|t|^{2},
\end{aligned}
$$

$R, T$ depend only on the normal component of wave vector $k_{\perp}$. This means that . $R, T$ are expressed as a function of $Q=2 k_{\perp}=4 \pi / \lambda_{\perp}\left(\lambda_{\perp}=\lambda / \sin \theta_{0}\right)$.

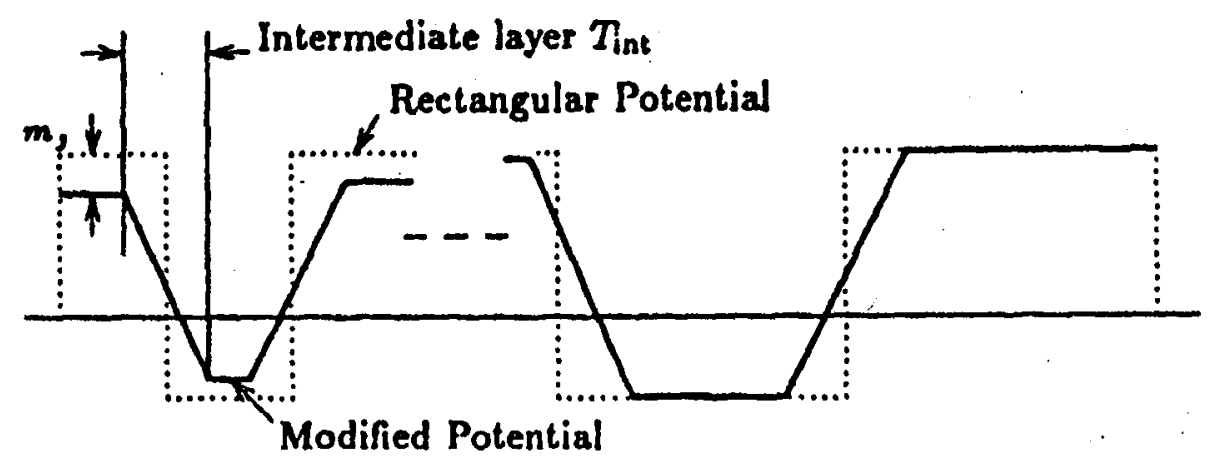

Fig. 2. Schematic representation of the modified optical potential. The reduction of potential's height and the smoothing at the potential boundary are introduced.

In practical calculations the modified potentials are used considering the roughnesses at the boundaries as shown in Fig. 2 [13]. And also reduction of density at evaporation is also taken into account.

\section{Monochromators and polarizing monochromators}

When thicknesses of layers are equal, neutron waves with the same incident angle are reflected at every boundary of the layers coherently like in the case of the Bragg reflection. Multilayer neutron mirrors consisting of alternating parallel layers of two substances of equal thickness are used as monochromators and polarizing monochromators. Figure 3 shows the example of the polarizing monochromator consisting of $\mathrm{Fe}_{50} \mathrm{Co}_{50} / \mathrm{V}$ of which the interplanar spacing is designed to be 


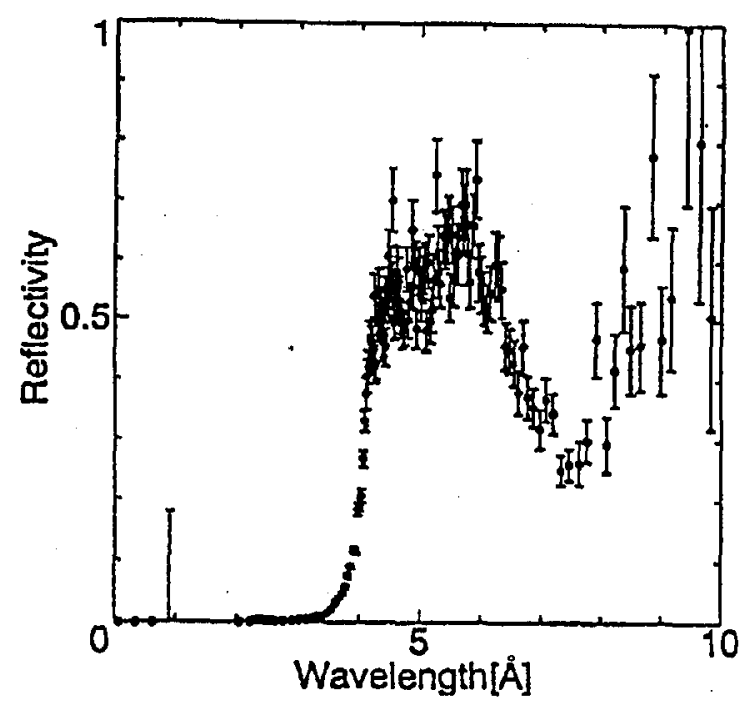

Fig. 3. Reflectivity of the $\mathrm{Fe}-\mathrm{Co} / \mathrm{V}$ polarizing monochromator. Polarization is $85 \%$ for $6.5 \AA$ neutrons of $5 \mathrm{~mm}$ in beam width.

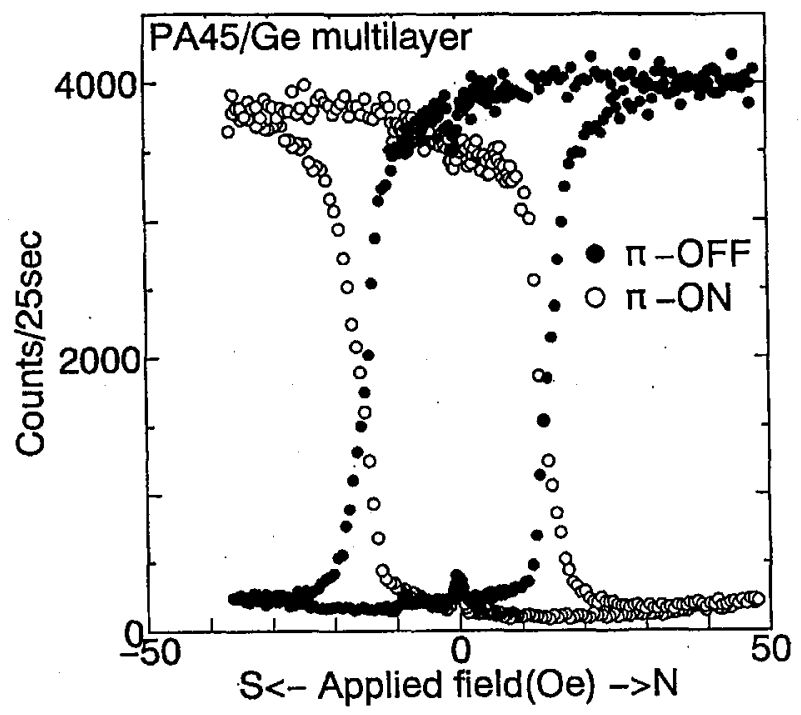

Fig. 4. Magnetization history of a permalloy/Ge polarizing monochromator.

$180 \pm 45 \AA$. This multilayer polarizing monochromator reflects polarized neutrons of $9 \AA$ with the full width of about $2 \AA$ at half maximum at the glancing angle of $1 / 40 \mathrm{rad}[27]$.

Recently we have developed the polarizing mirror working under very low external magnetic field consisting of permalloy/Ge multilayer. This polarizing mirror functions even under the zero magnetic field as shown in Fig. 4 [28]. 


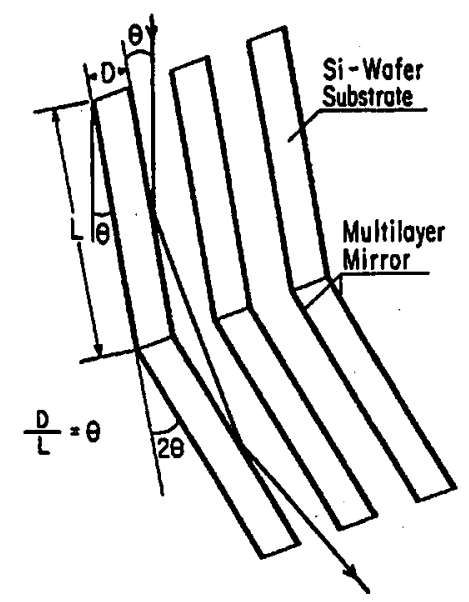

Fig. 5. Schematic representation of the double reflection multilayer monochromator of a Soller type.

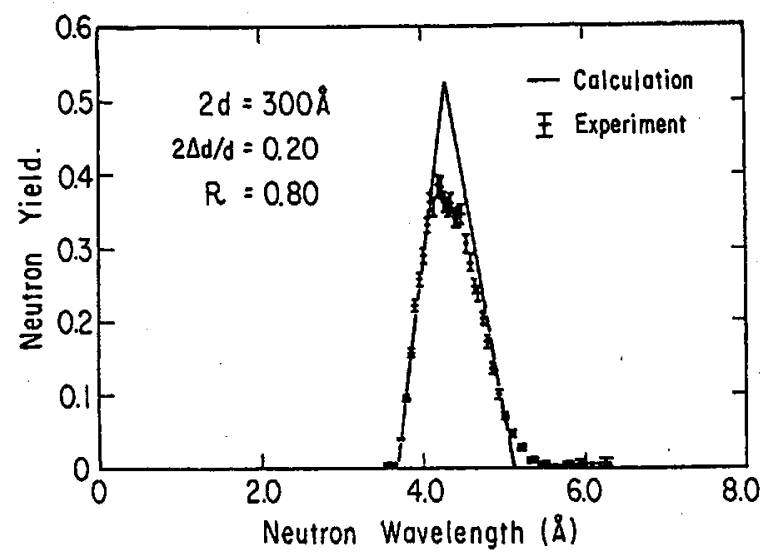

Fig. 6. Neutron spectra obtained by the double reflection monochromator.

Figure 5 depicts the schematic structure of the monochromator using the successive double reflection by two multilayer mirrors and Fig. 6 shows the experimental result [23].

\section{Supermirrors}

A multilayer composed of layers whose thicknesses are varied gradually layer by layer reflects neutrons with a wide wavelength range. Reflectivities of $\mathrm{Ni} / \mathrm{Ti}$ supermirrors and that of a Ni mirror of $1000 \AA$ in thickness are shown in Fig. 7 [17]. Taking $d_{0}=500 \AA, d_{1}=200 \AA, \Delta=20 \AA$ and $a=-4.5$, it is found that the best distribution of layer thicknesses of the $\mathrm{Ni} / \mathrm{Ti}$ supermirror is given by Eq. (20)

$$
d_{j}-d_{j+1}=\Delta\left(\frac{d_{1}}{d_{j}}\right)^{a} \quad(j=1,2, \cdots)
$$




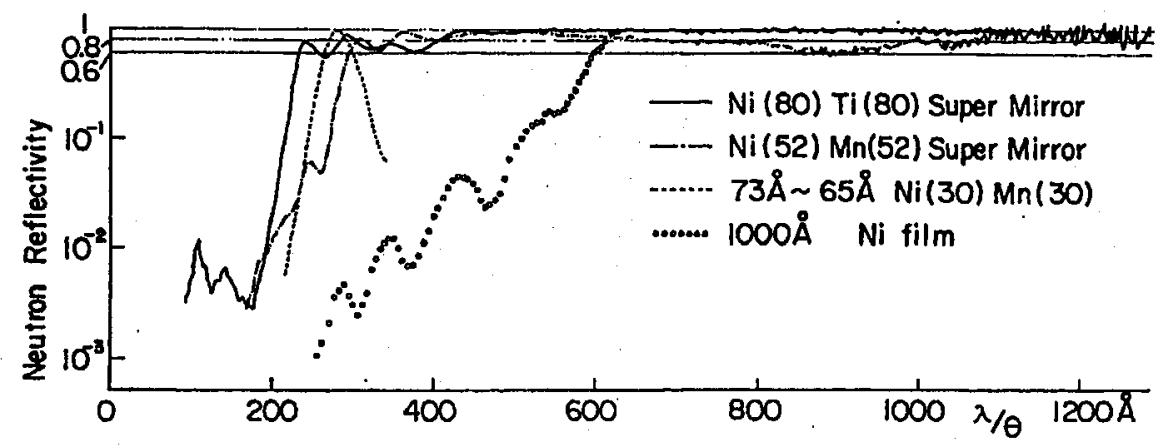

Fig. 7. Measured reflectivities of $\mathrm{Ni} / \mathrm{Ti}$ supermirror and Ni mirror of $1000 \AA$.

where $d_{j}$ is the thickness of the $j$-th layer, $\Delta$ defines the maximum decrease in layer thickness and $a$ means the parameter determining the distribution of thickness.

\section{Measuring methods of reflectivity and polarization}

The dependence of a reflectivity on the neutron wavelength is measured usually by two methods: time of flight (TOF) and $\theta / 2 \theta$ scanning methods. The time, $t$, a neutron takes to travel from a neutron source (a chopper) to a detector situated at a distance $\ell$, is

$$
t=\frac{m \ell}{h} \lambda
$$

From a distance of a flight path and a flight time, the dependence of a reflectivity on wavelength is obtained. A chopper is used to obtain "time zero" for the measurement by pulsing neutrons. The time resolution of the experiment is then controlled by the duration of neutron pulse and by the time resolution of the detector. The resolution of the wavelength $\Delta \lambda / \lambda$ is expressed as

$$
\left(\frac{\Delta \lambda}{\lambda}\right)^{2}=\left(\frac{\Delta t}{t}\right)^{2}-\left(\frac{\Delta \ell}{\ell}\right)^{2}
$$

where $\Delta t$ means the pulse duration and channel width of time analyzer, and $\Delta \ell-$ the error of flight path including detector thickness. The $\theta / 2 \theta$ scanning method is accomplished by Bragg reflection. A well-collimated neutron beam is allowed to be incident on a sample mirror and only neutrons of a given wavelength are reflected depending on the incidence angle. By changing the angle of incidence, neutrons of different wavelengths are reflected and with successive steps the intensity of spectrum is obtained. The Bragg relation between wavelength, the reflection angle $\theta_{\mathrm{B}}$ and the interplanar spacing $d$ of the multilayer is

$$
\lambda=2 d \sin \theta_{\mathrm{B}} .
$$

The wavelength resolution is

$$
\Delta \lambda / \lambda=\cot \theta_{\mathrm{B}} \Delta \theta_{\mathrm{B}} .
$$

In these methods, typical resolutions of wavelength are in the range of $1-5 \%$. Figure 8 shows the examples of the reflectivities measured. 


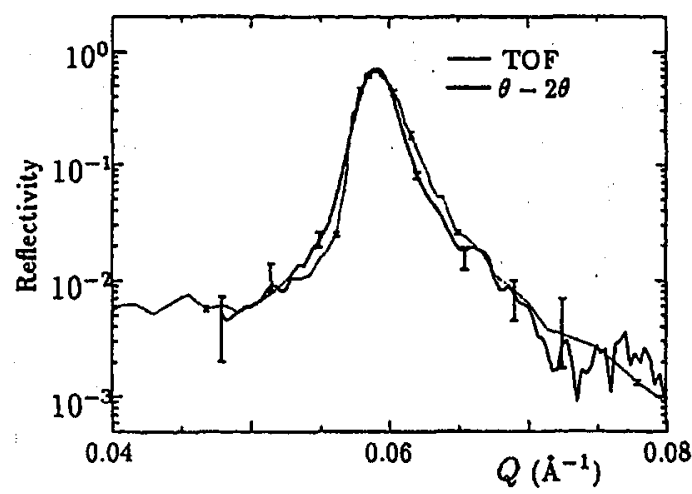

Fig. 8. Neutron reflectivities of $\mathrm{Ni} / \mathrm{Ti}$ monochromator of 100 layers measured by TOF and $\theta / 2 \theta$ scanning methods.

The practical polarization $P$ of neutron beam with a polarizing monochromator is defined as

$$
P=\frac{N_{\text {off }}-N_{\text {on }}}{N_{\text {off }}+N_{\text {on }}}
$$

where $N_{\text {off }}, N_{\text {on }}$ are the counting rates of neutrons reflected from the analyzing mirror in the case of $\pi$-flipper off and $\pi$-flipper on, respectively. The experimental reflectivity is defined as follows:

$$
R=\frac{N_{\text {ref }}}{N_{\text {ref }}+N_{\text {tr }}}
$$

where $N_{\text {ref }}$ is the counting rate of neutrons reflected from the analyzing mirror and $N_{\mathrm{tr}}$ that of neutrons transmitted through the mirror. The polarization value depends on a measuring instrument and system, especially the flipping ratio of $\pi$-flipper. The flipping ratio of a radio-frequency $\pi$-flipper is usually high compared with that of Mezei-type.

\section{Neutron guide tubes}

A neutron guide tube makes good use of the total reflection at the surface of materials. Material with large $b_{\mathrm{N}} \rho$ has an advantage for a neutron guide tube, since a critical angle is large. A float glass with a smooth surface or a silicon wafer is used as a substrate of a mirror to avoid microscopic unevenness and macroscopic undulations. Mirrors are fabricated by a vacuum evaporation or sputtering method. The neutron guide tube guides neutrons without loss of intensity to a distant place by arranging mirrors with a definite curvature. In the case of a curved guide tube which does not look through the neutron source, fast neutrons and $\gamma$ rays as noises are removed. Figure 9 describes the two kinds of neutron trajectories, garland and zigzag reflections.

Let us take $\theta^{*}$ as the maximum inciclent angle possible in the garland reflection. Refering to Fig. 9, the following relation is obtained:

$$
\sin \theta^{*}=\frac{L^{*}}{2\left(\varrho+d_{\mathrm{g}}\right)}
$$




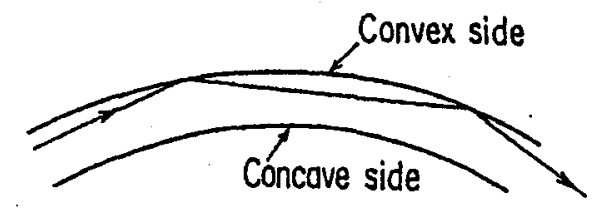

Gorland reflections

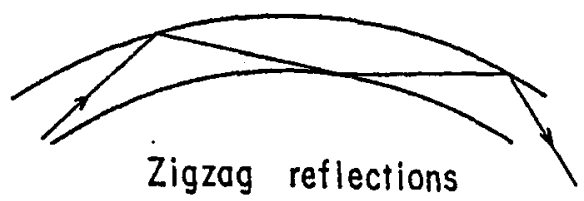

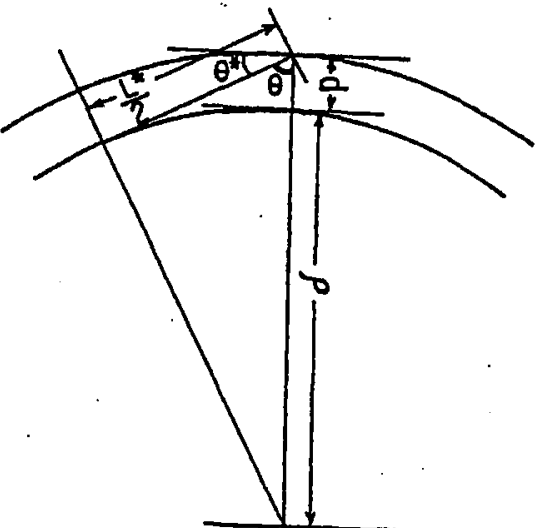

Fig. 9. Schematic neutron trajectories in a neutron guide tube and characteristic parameters for a guide tube.
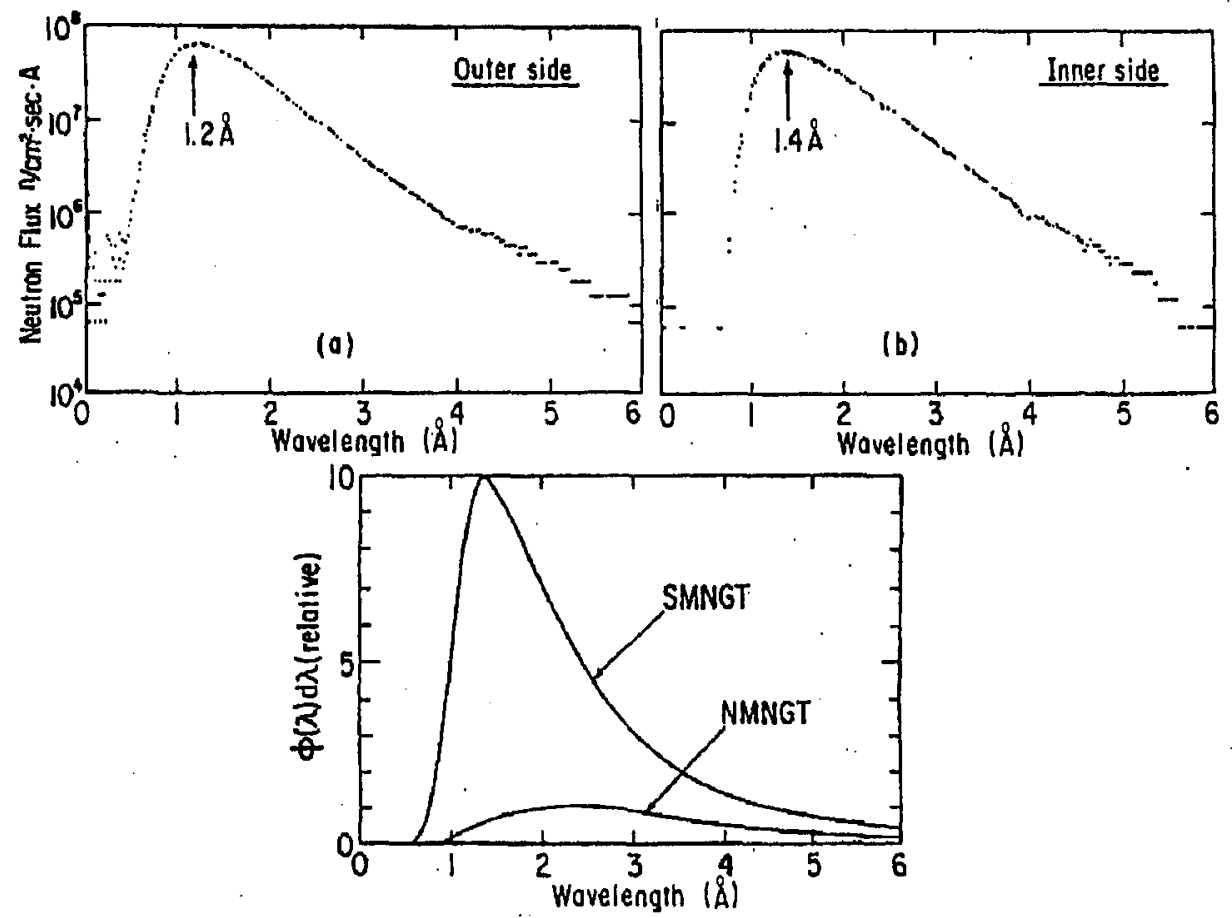

Fig. 10. Neutron spectra of KUR neutron guide tube; outerside (a) and innerside (b) of the guide. NMNGT means $\mathrm{Ni}$ mirror guide and SMNGT supermirror guide tube.

$$
L^{*}=2 \sqrt{2 \varrho d_{\mathrm{g}}+d_{\mathrm{g}}^{2}}
$$

where the characteristic path length, $L^{*}$, is the distance in which a neutron with the incidence angle $\theta^{*}$ reflects once and travels to the next reflecting place, $\varrho-$ 
the radius of curvature of the guide tube and $d_{\mathrm{g}}$ - the width of the guide tube. Taking into account that $\theta^{*} \ll 1, \varrho \gg d_{\mathrm{g}}$,

$$
\begin{aligned}
& \theta^{*} \simeq \sqrt{2 d_{\mathrm{g}} / \varrho}, \\
& L^{*} \simeq \sqrt{8 \varrho d_{\mathrm{g}}} .
\end{aligned}
$$

Thus the condition which neutrons with $\theta^{*}$ could pass through the guide tube by the total reflection is given by

$$
\theta^{*} \leq \theta_{\mathrm{c}}=\lambda \sqrt{b_{\mathrm{N}} \rho / \pi}
$$

The characteristic wavelength of the guide tube is defined as

$$
\begin{aligned}
& \lambda^{*}=\lambda_{1} \sqrt{2 d_{\mathrm{g}} / \varrho}, \\
& \lambda_{1}=\sqrt{\pi /\left(\rho b_{\mathrm{N}}\right)}=\lambda / \theta_{\mathrm{c}} .
\end{aligned}
$$

The length of a practical guide tube is a little bit longer than the $L^{*}$ to decrease fast neutrons and $\gamma$ rays. Decide the $L$ and $\lambda^{*}$ firstly. Then the structure of the guide tube $\left(\varrho, d_{\mathrm{g}}\right)$ is determined. A supermirror neutron guide tube makes use of the characteristic wave length ( $290 \AA$ for a Ni/Ti supermirror) shorter than that of $\mathrm{Ni}$ mirror $(580 \AA)$ and extracts neutrons of a shorter wavelength.

Figure 10 shows neutron spectra of KUR neutron guide tube.

\section{Neutron reflectometer}

Neutron refractive indices depend on kinds of isotopes or species of elements and their distribution. By measuring neutron reflectivity which depends on refractive indices in layers, we could understand the distribution of substances in a sample. Such an instrument is called a neutron reflectometer. Figure 11 shows the neutron reflectometer installed at C3-1-2 cold neutron guide tube of JRR-3M of JAERI [23].

Figure 12 shows one example of reflectivities measured by this reflectometer [29]. The sample is a ${ }^{6} \mathrm{Li} / \mathrm{Ti}$ multilayer neutron converter for the solid UCN detector working at liquid helium temperature. The result implies that the neutrons of wavelengths around $1500 \AA$ could be detected by the UCN detector. We used the microneutron interferometer (MINI) consisting of a thin Pd film sandwiched by a pair of $\mathrm{Ni} / \mathrm{Ti}$ multilayer mirrors to determine the hydrogen content in the evaporated palladium film. Two multilayer mirrors are semitransparent for a neutron and a neutron wave reflected from these mirrors interferes as shown in Fig. 13.

$$
\begin{aligned}
& \text { A phase difference is expressed approximately as } \\
& \phi=n_{\mathrm{Pd}} D Q
\end{aligned}
$$

where $n_{\mathrm{Pd}}$ is the refractive index of the palladium, $D-$ the thickness of palladium layer, $Q$-- the momentum transfer of neutron. Figure 14 shows neutron reflectivities from the MINI at 0, 1 and 2 atm liydrogen atmosphere. From this results the ratios $\mathrm{H} / \mathrm{Pd}$ are evaluated as $0.43 \pm 0.03$ and $0.55 \pm 0.03$ for 1 and $2 \mathrm{~atm}$, respectively. 


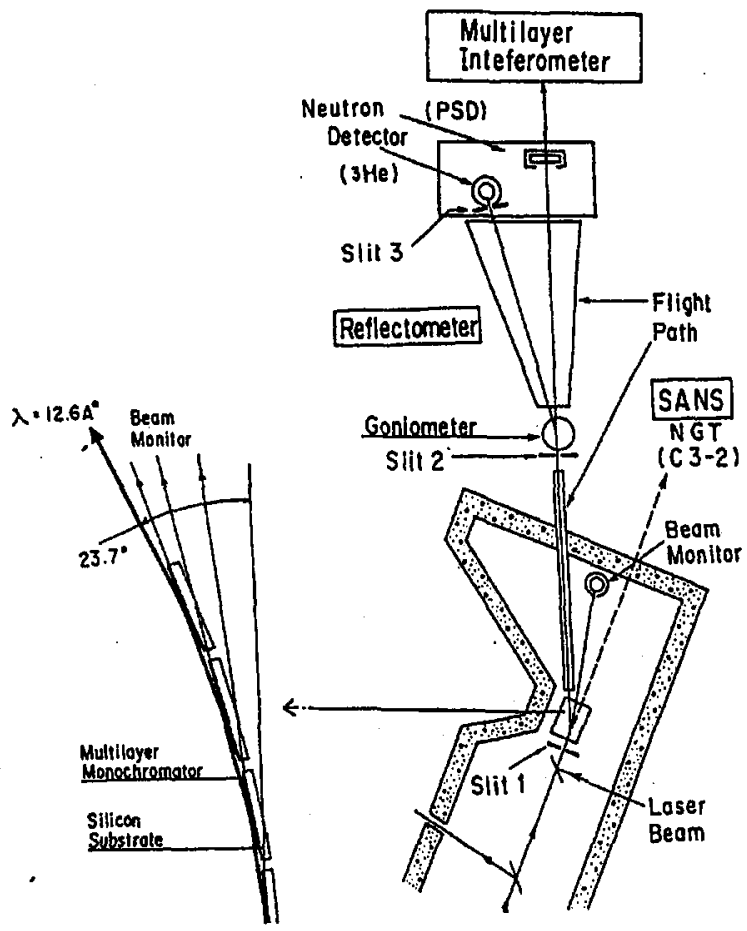

Fig. 11. The neutron reflectometer installed at C3-1-2 cold neutron guide tube of JRR-3M of JAERI. Neutrons are monochromatized and bent by four successive Bragg reflections from the quadri-polygon monochromator.

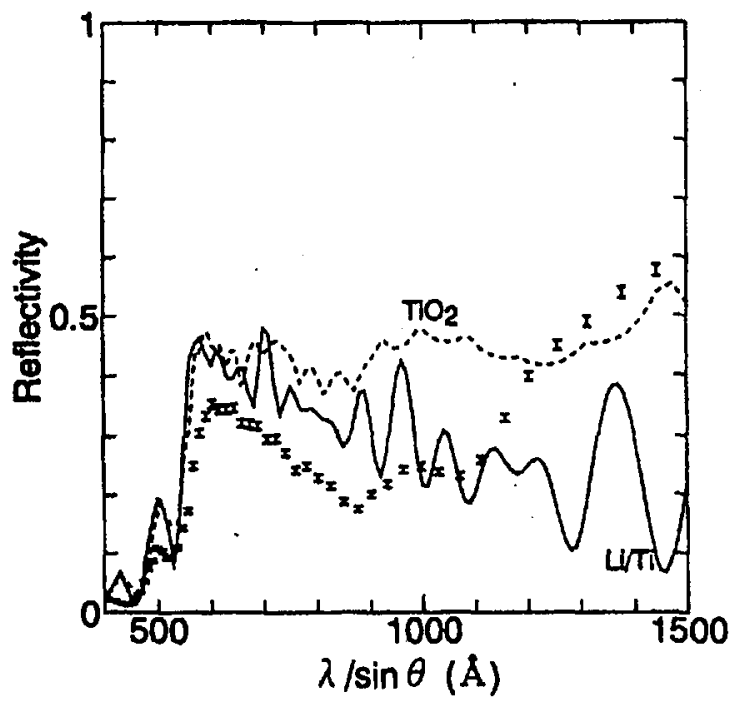

Fig. 12. The reflectivities of ${ }^{6} \mathrm{Li} / \mathrm{Ti}$ multilayer as a function of $\lambda / \sin \theta$. The curve with error bars represents the measured results. 


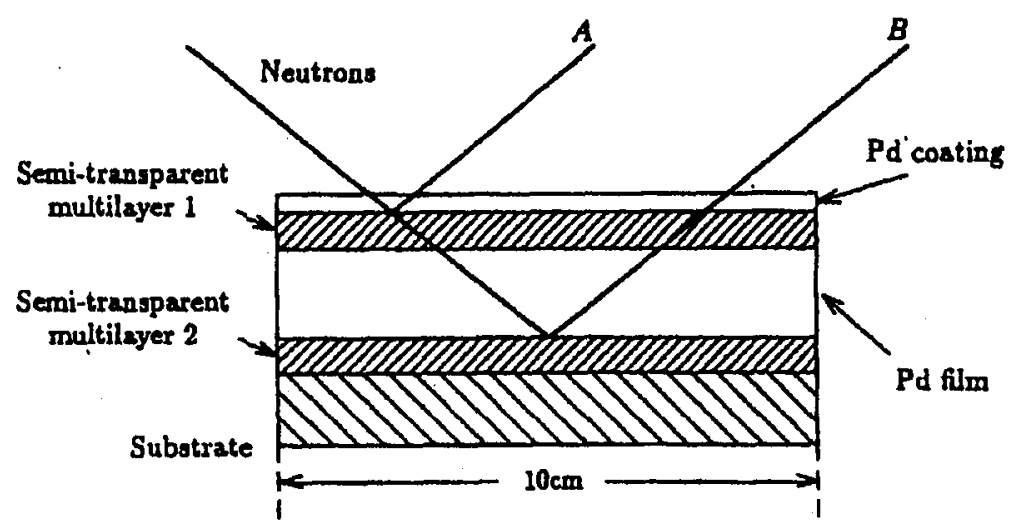

Fig. 13. The structure of a microneutron interferometer. A pair of Ni/Ti semitransparent layers sandwiches a palladium layer.

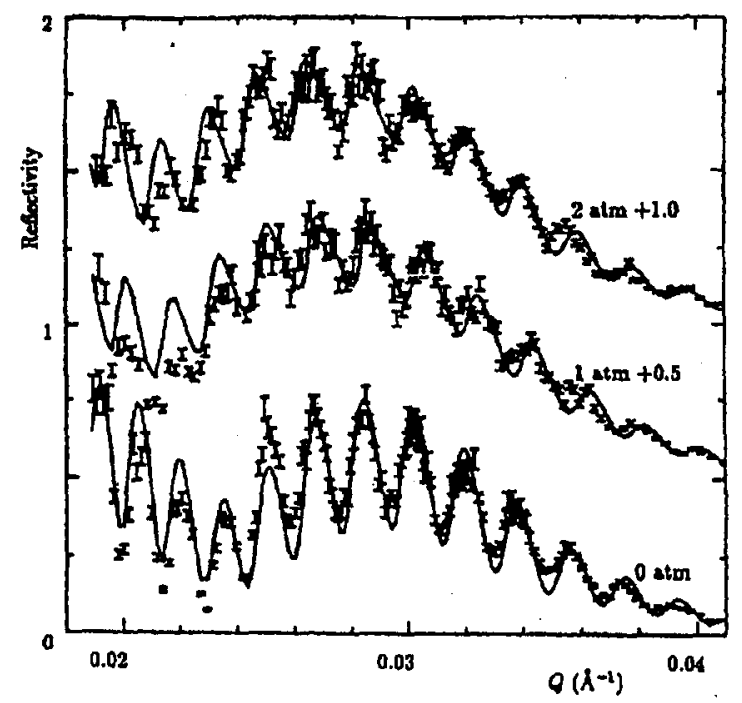

Fig. 14. Neutron reflectivities from a microneutron interferometer (MINI) at 0,1 , and 2 atm hydrogen atmosphere. The solid lines express the calculated results assuming the ratios of $\mathrm{H} / \mathrm{Pd}$.

\section{Acknowledgments}

I would like to thank our colleagues, especially Drs. T. Ebisawa, S. Tasaki, M. Hino, belonging to Neutron Science Division of KUR who have made all kinds of contributions. This work was partially supported by Grant-in-Aid for Scientific Research of the Ministry of Education of Japan under the program numbers 04244103 and 08404014.

\section{References}

[1] E. Fermi, W.H. Zinn, Phys. Rev. 70, 103 (1946).

[2] V.F. Turchin, Slow Neutrons, Sivan Press, Jerusalem 1965. 
[3] D.J. Hughes, Neutron Optics, Interscienc Pub. Inc., 1954.

[4] M.L. Goldberger, F. Seitz, Phys. Rev. 71, 294 (1947).

[5] I.I. Gurevich, L.V. Tarasov, Low Energy Neutron Physics, North Holland Pub. Co., Amsterdam 1968.

[6] V.F. Sears, Neutron Optics, Oxford University Press, New York 1989.

[7] H. Maier-Leibnitz, T. Springer, Reactor Sci. Tech. 17, 217 (1963).

[8] S. Okamoto, T. Akiyoshi, T. Ebisawa, S. Yamada, S. Mitani, F. Yoshida, T. Kawai, N. Achiwa, Neutron Guide Tube at KUR (1974) (in Japanese).

[9] T. Akiyoshi, T. Ebisawa, T. Kawai, F. Yoshida, M. Ono, S. Tasaki, S. Mitani, T. Kobayashi, S. Okamoto, J. Nucl. Sci. Technol. 1, 1 (1993).

[10] R. Pynn, Rev. Sci. Instr. 55, 837 (1984).

[11] S. Yamada, T. Ebisawa, N. Achiwa, T. Akiyoshi, S. Okamoto, Ann. Rep. Res. Reactor Inst. Kyoto Univ. 11, 8 (1978).

[12] T. Ebisawa, N. Achiwa, S. Yamada, T. Akiyoshi, S. Okamoto, J. Nucl. Sci. Tech. 16, 647 (1979).

[13] S. Tasaki, J. Appl. Phys. 71, 2375 (1992).

[14] S. Tasaki, T. Ebisawa, T. Akiyoshi, T. Kawai, S. Okamoto, Nucl. Instrum. Methods $A$ 355, 501 (1995).

[15] T. Kawai, T. Ebisawa, S. Tasaki, T. Akiyoshi, M. Hino, N. Achiwa, Y. Otake, H. Funahashi, J. Phys. Soc. Jpn. Suppl. 65, 230 (1996).

[16] B.P. Schoenborn, D.L.D. Casper, O.F. Lammerer, J. Appl. Cryst. 7, 508 (1974).

[17] T. Ebisawa, S. Tasaki, T. Akiyoshi, N. Achiwa, S. Okamoto, Ann. Rep. Res. Reactor. Inst. Kyoto Univ. 23, 46 (1990).

[18] J.W. Lynn, J.K. Kjems, L. Passell, A.M. Saxena, B.P. Schoenborn, J. Appl. Cryst. 9, 454 (1976).

[19] F. Mezei, Commun. Phys. 1, 81 (1976).

[20] G.P. Felcher, Phys. Rev. B 24, 1595 (1981).

[21] E. Bouchaud, B. Farnoux, X. Sun, M. Daoud, G. Jannink, Europhys. Lett. 2, 315 (1986).

[22] J. Penfold, R.K. Thomas, J. Phys., Condens. Matter 2, 1369 (1990).

[23] T. Ebisawa, S. Tasaki, Y. Otake, H. Funahashi, K. Soyama, N. Torikai, Y. Matsushita, Physica B 213\&214, 901 (1995).

[24] S. Tasaki, T. Kawai, T. Ebisawa, J. Appl. Phys. 78, 2398 (1995).

[25] T. Kawai, M. Utsuro, Y. Maeda, T. Ebisawa, T. Akiyoshi, H. Yamaoka, S. Okamoto, Nucl. Instrum. Methods A 276, 408 (1989).

[26] P.A. Krupchitsky, Fundamental Research with Polarized Slow Neutrons, Springer-Verlag, Tokyo 1987.

[27] T. Kawai, S. Tasaki, T. Ebisawa, Y. Eguchi, M. Hino, N. Achiwa, H.M. Shimizu, Ann. Rep. Res. Reactor Inst. Kyoto Univ. 27, 235 (1994).

[28] T. Kawai et.al., to be published.

[29] T. Kawai, T. Ebisawa, S. Tasaki, H.M. Shimizu, Nucl. Instrum. Methods A 378, $561(1996)$. 